\title{
Corela
}

Cognition, représentation, langage

HS-8 | 2010

L'interpellation

\section{L'interpellation : interjections et appellatifs. Une approche contrastive italien/français}

\section{Carlotta Cini}

\section{(2) OpenEdition}

1 Journals

Édition électronique

URL : http://journals.openedition.org/corela/1698

DOI : $10.4000 /$ corela. 1698

ISSN : 1638-573X

Éditeur

Cercle linguistique du Centre et de l'Ouest - CerLICO

\section{Référence électronique}

Carlotta Cini, «L'interpellation : interjections et appellatifs. Une approche contrastive italien/français », Corela [En ligne], HS-8 | 2010, mis en ligne le 11 novembre 2010, consulté le 21 décembre 2020. URL http://journals.openedition.org/corela/1698; DOI : https://doi.org/10.4000/corela.1698

Ce document a été généré automatiquement le 21 décembre 2020.

\section{(c) (i) (2)(2)}

Corela - cognition, représentation, langage est mis à disposition selon les termes de la licence Creative Commons Attribution - Pas d'Utilisation Commerciale - Partage dans les Mêmes Conditions 4.0 International. 


\title{
L'interpellation : interjections et appellatifs. Une approche contrastive italien/français
}

\author{
Carlotta Cini
}

\section{Introduction}

1 Selon la littérature intéressée, les interjections et le système de l'adresse sont deux catégories séparées. Les formes d'adresse sont définies par Braun (1988) « [the] speaker's linguistic reference to his/her collocutor(s) $»^{1}$ et comprennent seulement les verbes, les noms et les pronoms. Les interjections sont généralement définies comme des éléments linguistiques qui servent à établir un contact. Selon nous, au contraire, certaines interjections font partie du système de l'adresse; on pourrait dire que, dans certains cas, on a affaire à des hybrides, qui montrent une étroite liaison entre l'interjection et l'appellatif: il s'agit de ce que nous appelons ici les interjections vocatives.

2 L'approche théorique qui est à la base de cette recherche est pragmatiqueinteractionnelle; bien que la conversation filmique ne soit pas spontanée mais fictive, en effet, elle reprend et reflète les dynamiques de la conversation naturelle. En particulier, je me suis appuyée sur la théorie de la politesse (Brown-Levinson, 1983 et KerbratOrecchioni, 1994) et le concept de considération (André-Larochebouvy, 1984); pour ce qui concerne la pragmatique contrastive nous avons considéré surtout les travaux de Wierzbicka (1985) et Blum-Kulka (1989); en traduction, nous avons employé le modèle pragmatique-communicatif de Halliday et Hatim-Mason (1990). Pour les termes d'adresse les approches de Traverso (1996) et Braun (1988) ont été fondamentales; pour les interjections nous avons suivi principalement les études de Bazzanella (1994) et Serianni (2000).

3 Nous avons aussi tenu en compte la dimension «orale » des éléments linguistiques analysés, leur sens à l'intérieur de la conversation ne pouvant pas être séparé des 
données paralinguistiques (vitesse d'élocution, intensité vocale, hauteur de la voix) ni des données non verbales (mimiques, posture, estes, regard, proxémique ${ }^{2}$ ). Le corpus est constitué de trois films italiens sous-titrés en français: Sciuscià, (Vittorio De Sica, Italia, 1946), Mamma Roma (Pier Paolo Pasolini, Italia 1962), Respiro (Emanuele Crialese, Italia, 2003).

L'étude est organisée en trois parties: le première concerne la définition et l'explication du concept d' "interjection vocative», que nous analysons, au sein des stratégies d'interpellation, d'un point de vue linguistique et pragmatique. La deuxième est consacrée au rôle de ces particules à l'intérieur des films analysés, à travers une sélection d'extraits, et aux modifications produites dans les versions françaises; une analyse quantitative des données a été réalisée. Enfin, nous proposons des alternatives censées éviter de perdre complètement, dans la version cible, la dimension interpersonnelle de la version source.

Légende:

6 Symboles prosodiques utilisés:

$7 \uparrow$ intonation montante

$8 \downarrow$ intonation descendante

9 : pause brève

10 :: pause longue.

11 Abréviations:

12 IV: interjection vocative

13 ST: sous-titres

14 VO: version originale

15 ST: version sous-titrée

16 FFA: Face Flattering Act.

17 FTA: Face Threatening Act.

\section{Interjections et système de l'adresse}

\subsection{Les interjections}

Les interjections se caractérisent d'abord par leur caractère d'instantanéité expressive, une dimension qui a toujours été mise en relief en dépit de leur rôle au niveau de l'interaction discursive. Particules multifonctionnelles, leur sens dépend fortement des caractéristiques prosodiques, qui ont une fonction de désambiguïsation. Elles sont employées surtout dans des situations informelles et dans des relationèmes (KerbratOrecchioni, 1994) de type familier ou amical. Une autre caractéristique intéressante est le fait qu'elles ont la capacité de réaliser le sens d'une phrase entière (par exemple « ouf! », en français, est une marque de soulagement qui peut signifier, sans avoir besoin d'autres éléments linguistiquement structurés, « je suis soulagé »)

Grâce à ces particularités, les interjections ont été longtemps considérées comme l'expression spontanée et non structurée d'une réaction impromptue et instinctive de l'esprit. Bazzanella (1966) leur attribue le caractère de «cancellabilità semantica » (234), selon lequel elles peuvent être effacées sans que le sens en ressente. Ce point de vue est 
partagé par Battaglia (1977) qui ajoute que ces particules sont dépourvues de toute fonction syntaxique.

Ducrot, en 1980, pose les bases d'une interprétation différente des interjections, qu'il considère comme des jeux de langage qui permettent aux locuteurs d'adopter des attitudes, de jouer des rôles, parmi lesquels, par exemple, informer l'interlocuteur d'un état émotionnel.

21 Mais il faudra attendre les études de Sacks, Schegloff et Jefferson (1974) pour que ces particules se voient attribuer un rôle structurel dans la communication: il les présente pour la première fois comme des TCU (Turn Constructional Units), des unités qui concourent à la construction des tours de parole.

Dans les années suivantes un véritable statut à l'intérieur du domaine de l'interaction commence à leur être attribué (Costa, 1981): on s'aperçoit que les interjections sont des unités soumises à des conditions précises, qui varient d'une langue à l'autre et d'une situation de communication à l'autre.

En 1985, Wierzbicka entérine cette nouvelle interprétation en ajoutant à la fonction/ catégorie émotive, généralement reconnue comme la seule fonction des interjections, deux autres fonctions: volitive et cognitive. L'introduction de ces deux catégories revient à attribuer à ces particules une fonction conversationnelle qui intègre celle exclusivement expressive/émotive qui leur était attribuée précédemment.

\subsection{Le système de l'adresse}

Selon Traverso (1996), lesystème de l'adresse inclut les pronoms d'adresse et les termes d'adresse (les appellatifs/noms d'adresse à travers lesquels le locuteur s'adresse au récepteur).

Bien que la première fonction des termes d'adresse soit l'identification, ces particules ont aussi d'autres fonctions d'ordre pragmatique. En particulier, elles servent à attirer l'attention de l'interlocuteur, à stimuler sa participation à l'échange - fonction que André-Larochebouvy $(1984,160)$ appelle "considération" -, à l'établissement du jeu des distances sociales, à travers la révélation de l'intention du locuteur dans la communication, l'expression de l'opinion qu'il a de son interlocuteur, etc. En particulier, elles semblent avoir un rôle important dans les stratégies de politesse: le choix du terme d'adresse peut, selon le contexte, constituer un acte flatteur, ou menaçant (KerbratOrecchioni, 1994).

Par ailleurs, elles agissent au niveau de la «mécanique de la conversation»; elles sont déterminées par et rendent compte du fonctionnement des tours de parole, de la relation entre les interlocuteurs (formelle, informelle, etc.), de la négociation des identités et des distances sociales, du récepteur auquel on s'adresse (récipient design: orientation en fonction du récepteur), des intentions des interlocuteurs, de la société d'appartenance des locuteurs, de la subjectivité du discours (l'auto-implication du locuteur et la dimension de tension ou de modulation qui s'ensuit.

Dans la fiction filmique, l'appellatif devient, comme le dit Roland Barthes, (1972) « un signe volumineux, un signe toujours gros d'une épaisseur touffue de sens » (121).

En relation avec ces fonctions pragmatiques, le système de l'adresse varie sensiblement selon différents paramètres. D'abord, selon la société d'appartenance des locuteurs: 
certains « ethnolectes conversationnels » (Kerbrat-Orecchioni, 1990, 63) sont caractérisés par une utilisation des appellatifs plus fréquente, un éthos à dominante égalitaire ${ }^{3}$ ou hiérarchique, etc.; deuxièmement, selon le récepteur auquel on s'adresse (recipient design: orientation en fonction du récepteur); enfin, selon l'intention du locuteur, notamment conflictuelle, harmonieuse, censée offenser son interlocuteur, le convaincre, le dissuader, etc.

Malgré leursdifférences, interjections et appellatifs ont plusieurs points en commun: ils ont tous les deux un fort caractère déictique, c'est-à-dire qu'ils nécessitent un contexte de référence immédiat et qu'ils ne peuvent concerner que le présent; au niveau diastratique, ils renvoient souvent à une origine sociale populaire; au niveau diaphasique, ils rendent compte d'une relation informelle et impliquent donc une distance réduite entre les interlocuteurs; ils peuvent exprimer le mépris, la colère, la menace, aussi bien que l'affection et la moquerie; ils peuvent établir une relation de parité (symétrique) ou de hiérarchie (asymétrique, où le récepteur est en position d'infériorité).

\subsection{Le interjections vocatives (IV)} exactement à l'intérieur du lien entre ces deux catégories linguistiques et relèvent de l'une et de l'autre. Il s'agit des interjections employées, dans la communication orale quotidienne, pour interpeller son interlocuteur («eh!», «oh!», «ehi!», etc.). Ces particules ont la forme des interjections mais la fonction des termes d'adresse: leur emploi même dans le processus de l'interpellation est régi par les mêmes paramètres qui régissent les appellatifs (Costa, 1981, 87-124). Parmi les interjections elles sont les moins iconiques, ce qui fait qu'elles changent sensiblement d'une langue à l'autre (Blum-Kulka, 1989), tout comme le système de l'adresse, et voire à l'intérieur d'une même langue selon les différentes variétés diatopiques. En particulier, pour ce qui concerne notre recherche, les IV choisies sont typiques de certains dialectes italiens (présents dans les films en question) et souvent ne trouvent pas de correspondant dans la langue italienne standard ; même leur fréquence d'emploi est beaucoup plus grande qu'en « italien non dialectal ».

Loin d'avoir une fonction exclusivement émotive, ces particules sont chargées d'une forte dimension conversationnelle et semblent jouer un rôle fondamental dans l'établissement des distances sociales et dans la dimension de la «considération». En outre, elles 
concourent à réaliser la dimension de tension ou de modulation (et donc mimétique ou agonale) de l'échange verbal: elles sont à considérer comme un moyen pour exprimer la subjectivité du discours, qui, selon Vion $(1992,247)$, peut atteindre toutes les parties du discours. Enfin, ces particules ont un rôle important dans le déroulement des stratégies de politesse entre les interactants, et concourent à rendre l'échange conflictuel ou harmonieux: l'interpellation est l'une des situations communicatives où les jeux concernant la «face » des interlocuteurs sont le plus clairement mis en évidence et où, par conséquent, les stratégies de politesse jouent un rôle central.

Les IV pourraient être confondues avec les connecteurs phatiques, mais elles s'en différencient sous plusieurs points de vue. D'abord, les connecteurs phatiques sont potentiellement toujours spéculaires, alors que les IV rendent compte d'une situation asymétrique: employées plus souvent par le locuteur que par le récepteur, elles peuvent être employées par un locuteur en position dominante envers un récepteur subalterne mais le contraire est beaucoup plus rare. En outre, elles ne concernent pas seulement le texte, mais incluent aussi les participants, comme on le voit dans ces exemples tirés de Respiro et Sciuscià:

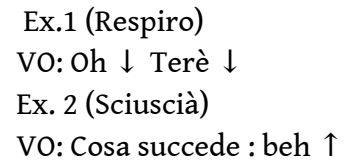

Enfin, elles renvoient à une relation informelle entre les interlocuteurs et souvent à une origine sociale populaire, fonction que le connecteur phatique n'a pas.

À l'intérieur des IV, il est nécessaire de faire une distinction entre les particules vocatives autonomes et non-autonomes. Afin de les distinguer, nous avons pris en considération principalement trois paramètres: leur possibilité de remplacer un terme d'adresse, l'importance de la dimension prosodique (la nécessité d'être accompagnées par le contact visuel et/ou gestuel), leur séparation du reste de l'énoncé. Dans cet article, nous analysons quatre IV (« ahó » et « oh » pour les IV autonomes et « a' » et « o' » pour les IV non autonomes) qui appartiennent aux dialectes parlés dans les films analysés (le napolitain, le sicilien et le romain).

Les IV autonomes peuvent tout à fait remplacer un terme d'adresse:

Ex. 3 (Mamma Roma)

VO: ahó $\downarrow$ Vie' ccà $\downarrow$

Elles sont toujours accompagnées du contact visuel entre les interlocuteurs, d'une intonation descendante et d'un soulèvement de la voix, souvent d'un geste de la main ou de la tête. Elles sont toujours suivies d'une pause, plus ou moins longue, qui les sépare du reste de l'énoncé.

Les IV autonomes ( $\mathrm{a}^{\prime}$ ", dans notre cas) au contraire, ne peuvent pas remplacer un terme d'adresse; elles sont toujours accompagnées d'un appellatif, souvent de parenté (Ex.Respiro: a' pa' (" papà »), d'un nom commun ou de personne abrégé (souvent une seule lettre: Mamma Roma: a' E' (de « Ettore ») / a' more' (de: " moretto »), d'un surnom ( Sciuscià: a' Napoli / Mamma Roma: a' turco) d'un hypocoristique, dont dépend leur valeur. Elles ne sont pas forcément accompagnées du contact visuel, car la fonction d'identification est déjà accomplie par l'appellatif qu'elles accompagnent; elles ne sont pas suivies d'une pause.

41 Ces particules, censées établir un contact avec le récepteur, sont très fréquentes dans les dialectes de l'italien, et changent d'une variété à l'autre; cela est dû aussi au fait que 
l'italien est une langue high-kinesic, où les interlocuteurs tendent à raccourcir les distances qui les séparent. Le français, langue à éthos low-kinesic, c'est-à-dire où les interlocuteurs tendent à garder la distance (linguistique et proxémique) entre euxmêmes, présente ces IV plus rarement.

\section{L'analyse}

\subsection{Présence des IV dans les sous-titres français} généralement éliminées. Voyons à ce propos le tableau suivant:

\begin{tabular}{|l|l|l|l|l|}
\hline Film & Appellatifs & $\begin{array}{l}\text { Interjection vocative } \\
\text { autonome }\end{array}$ & $\begin{array}{l}\text { Interjection vocative } \\
\text { non autonome }\end{array}$ & Sous-titres \\
\hline Mamma Roma & 186 & 38 & 77 & 75 \\
\hline Respiro & 96 & 9 & 10 & 6 \\
\hline Sciusciá & 96 & 6 & 24 & 0 \\
\hline
\end{tabular}

Cette analyse quantitative nous montre que, dans les films analysés, les appellatifs apparaissent dans les versions françaises à peu près la moitié des fois, les IV autonomes très rarement et les IV non-autonomes jamais. Si, comme l'observe Hatim-Mason (1990), dans les ST le système de l'adresse est sous représenté, les interjections vocatives le sont encore moins. français l'emploi de ces particules emploi est beaucoup moins fréquent qu'en italien, ce qui est à mettre en relation avec la différence entre l'éthos low-kinesik des français et l'éthos high-kinesik des italiens. Par ailleurs, il n'existe en français autonome aucun correspondant de la particule non autonome " $\mathrm{a}$ ' »; toutefois dans certaines variétés diatopiques, comme le marseillais, on trouve l'IV « $\mathrm{O}^{\prime}$ » (« $\mathrm{O}^{\prime}$ Michel! »), qui a exactement la même utilisation que " $\mathrm{a}^{\prime}$ " .

La deuxième raison est constituée par les contraintes spatiales et temporelles imposées par la traduction filmique. Elles entraînent une réduction des dialogues qui s'effectue principalement à travers la suppression de tout ce qui concerne les fonctions phatique, émotive, les répétitions, les noms, les exclamations, les opérations de ritualisation du dialogue et les éléments du discours oral (ellipse, interjections, hésitations, etc.); en outre, à l'écrit, les interjections apparaissent surtout dans les textes qui veulent imiter la langue orale. L'interpellation est donc l'une des catégories les plus sacrifiées dans le sous-titrage filmique.

Enfin, il faut considérer la nature du dialogue filmique, où le langage est souvent complété (ou, au moins, à mettre en relation) par le contexte et par d'autres signes (le setting, les vêtements, etc.).

Il en résulte une réduction de la subjectivité des échanges, et une modification du jeu des distances sociales: cela montre d'un côté le rôle structural de ces particules au sein du système de l'interpellation, et de l'autre la nécessité de repérer, dans la pratique de la traduction filmique, les alternatives potentielles que la langue française offre pour compenser cette perte. 


\subsection{Conséquences de l'élimination des IV dans les sous-titres}

\subsubsection{La réduction de la dimension de familiarité et de camaraderie}

formelle pour le scène en question (en outre, elle constitue un acte performatif qui n'existe pas dans la Vo); mais ce qui nous intéresse ici est l'élimination de l'IV "a'", liée à l'appellatif de parenté abrégé "ma"'. Dans la Vo, l'IV sert, comme dans l'exemple précédent, à expliciter le relationème enfant-mère, ce qui est censé flatter la face positive $d u$ récepteur et renforcer le lien entre les interlocuteurs, afin d'obtenir une réponse positive. Dans la V. ST tout cela est complètement effacé; cela comporte aussi un effet de détachement chez le spectateur, à cause de la différence entre les ST et les images, où les données proxémiques et la gestuelle - déplacement en avant du torse vers l'interlocuteur, allongement des mains, contact visuel - des acteurs montrent une dimension de proximité et de familiarité.

Voyons d'autres exemples qui montrent la perte de la dimension de familiarité et camaraderie provoquée, dans la V.ST, par l'élimination des IV:

Ex. 6 (Respiro)

VO: O' Pi' $\downarrow$

VST: ------

son lit et déprimé à cause d'une crise conjugale, ne veut pas se lever; l'enfant, poussé par des amis de son père, essaye de le convaincre d'aller avec lui à la chasse aux lapins.

Déjà, le fait d'appeler l'interlocuteur par son nom (dans ce cas, le nom de parenté) typiquement dialectale et familiale de "papà") est précédé par la particule vocative " $a^{\prime}$ ", elle aussi dialectale et familiale; cette combinaison crée une dimension de familiarité, car elle établit une liaison entre interlocuteurs qui dérive non seulement de l'explicitation de leur lien de parenté mais aussi du fait que les personnages partagent la même variété linguistique. Grâce à tous ces éléments, et donc aussi à la présence de l'IV non autonome " $\mathrm{a}^{\prime}$ ", l'expression de la VO devient un Face-Flattering Act (FFA, acte flatteur), censé obtenir une réponse positive de la part du récepteur.

ans à peu près, emprisonné pour avoir vendu des couvertures américaines volées, mande à sa mère de lui pardonner:

Ex. 5 (Sciuscià)

VO: A' mà $\downarrow$ me perdoni mà $\uparrow$

VST: Maman, je te demande pardon.

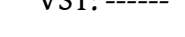



et une dame:

Ex. 11 (Sciuscià)

VO: Signo' :: dans les ST:

$$
\begin{aligned}
& \text { Ex. } 7 \text { (Respiro) } \\
& \text { VO: O' scià } \downarrow \\
& \text { VST: ----- }
\end{aligned}
$$
Mamma Roma:

Ici, Grazia, une femme, s'adresse à un ami de son mari, qu'elle appelle par son nom abrégé ("Pi'", abréviation de Piero) précédé par l'IV non autonome " $\mathrm{o}^{\prime}$ » (le correspondant sicilien du romain « $\left.a^{\prime} »\right)$, ce qui véhicule un sens de complicité et d'absolue informalité, effacé dans la V.ST par l'élimination de cette forme d'interpellation.

Dans les trois exemples qui suivent, les IV véhiculent une dimension de complicité et de camaraderie, qui n'est pas reproduite dans les respectives V. ST; dans les deux premiers, il s'agit de l'IV non autonome "a", très fréquente,comme on l'a vu dans le tableau, dans

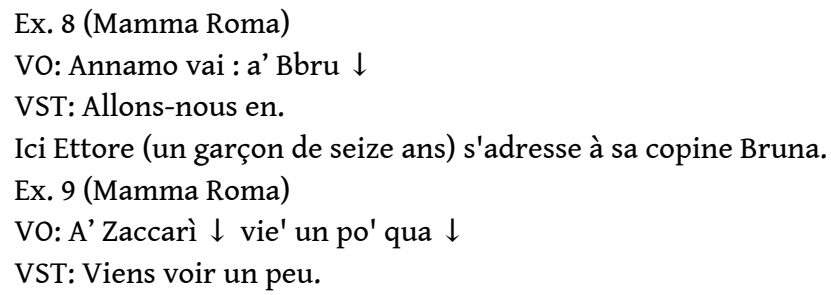

Dans cet extrait le dialogue se déroule entre Mamma Roma, la mère d'Ettore, prostituée, et Zaccaria, un souteneur.

Dans le dernier extrait, où une prostituée s'adresse à un client,enfin, l'élimination dans les ST touche l'IV autonome "ahó":

Ex. 10 (Mamma Roma)

VO: ahó : me sei simpatico $\downarrow$

VST: Tu es vachement sympa.

\subsubsection{La modification des distances sociales}

Une autre conséquence de l'effacement des IV dans les ST est la modification des distances sociales à l'intérieur d'un relationème.

relationèmes. Dans une situation formelle (enfant $\rightarrow$ adulte ou adulte $\rightarrow$ adulte) normalement l'IV n'est pas employée: dans Sciuscià, par exemple, on compte 23 honorifiques et titres sans I.V.

La scène dont l'exemple suivant est tiré se déroule au commissariat, entre le commissaire l s'agit d'un relationème adulte - adulte et, par ailleurs, en milieu institutionnel; comme on le voit, aucune IV n'est utilisée.

Dans le cas d'un relationème enfant-adulte, au contraire, les interjections vocatives sont beaucoup plus fréquentes: toujours dans Sciuscià, on trouve 21 noms propres/communs accompagnés par une I.V.: 
Ex. 12 (Sciuscià)

VO: Oè ragazzi :: guardate me

VST: Les gamins, regardez-moi.

\section{au sens linguistique du mot:}

Ex. 13 (Mamma Roma)

Vo: ahó : a' dritto $\downarrow$ mica ho messo ar monno un fijo per fallo diventà bburino $\downarrow$

VST: Je n'ai pas fait un fils pour [...]!

74 Cet appellatif est introduit ici par deux interjections vocatives, l'une autonome (" ahó »), l'autre non autonome («a'»). Bien que l'ironie soit réalisée principalement à travers l'appellatif, le fait que ce mot est accompagné des deux IV montre leur importance dans ce contexte; en outre, à travers leur force interpellative, ces IV augmentent l'intention ironique du locuteur, tout comme les comportements proxémiques (mouvement de la main, regard, rapprochement des sourcils, déplacement de la tête en avant) du locuteur. 


$$
\text { formule d'interpellation, l'expression sarcastique est accompagnée quand même d'une }
$$
IV, comme dans le cas suivant:

Ex. 16 (Mamma Roma)

VO: ahó $\downarrow$ Ma che hai perso la favella $\downarrow$

VST: $\mathrm{Tu}$ as perdu la parole?

81 Il s'agit toujours de Mamma Roma s'adressant à Ettore; le "ahó", qui contribue à augmenter l'intention moqueuse du locuteur n'est pas reproduite dans la V. ST, où la question posée est tout à fait neutre.

\subsubsection{Réduction de la dimension agressive et de l'expression du mépris}

D'après l'analyse, il semble que l'interjection vocative soit plus fréquemment reproduite dans les V. ST lorsqu'elle a une fonction agressive et menaçante; d'ailleurs, les appellatifs offensifs sont - eux-mêmes - reproduits plus souvent que les autres dans les VST. Voyons les deux exemples qui suivent:

Ex. 17 (Mamma Roma)

VO: ahó : Già m'hai stufato $\downarrow$

VST: Hé! Tu me les gonfles! 
ici d'une querelle entre deux jeunes gens, Ettore et Bruna; comme on le voit, l'IV autonome "ahó" est traduite par "hé" dans la V. ST. Voyons aussi l'exemple suivant, tiré toujours de Mamma Roma:

Ex. 18 (Mamma Roma)

VO: ahó : A carogna ma che sei comunista $\downarrow$

VST: Hé! Le salaud! T'es communiste? face positive de l'autre, est réalisée à travers l'appellatif "carogna" et deux IV. Dans la V. ST, l'IV non autonome n'est pas reproduite mais l'IV autonome et l'appellatif sont présents, ce qui est suffisant à rendre la dimension conflictuelle de la scène.

Pourtant, souvent même dans ce cas les IV sont effacées:

Ex. 19 (Sciuscià)

VO: Oh : Ce devi annà $\downarrow$

VST: Tu dois y aller.

Cette scène se déroule en prison, où un garçon menace un camarade; la menace du locuteur est réalisée surtout à travers les données paraverbales (voix haute et agressive) et proxémiques (rapprochement des sourcils, déplacement de la tête en avant, geste de la main) et l'IV "Oh". L'élimination de cette particule dans la V. ST comporte une diminution de la dimension conflictuelle de l'échange.

Dans l'extrait suivant, c'est une IV non autonome qui est éliminée:

Ex. 20 (Mamma Roma)

VO: a' pezza da piedi $\downarrow$ ::

VST: Salope.

Dans la V. ST l'offense est exprimée seulement par le mot « salope » alors que dans la VO l'appellatif offensif "pezza da piedi" (qui pourrait correspondre en français à « espèce de torchon ») est renforcé par l'IV “a'”, qui ajoute du mépris à l'offense, mis en valeuraussi par l'intonation descendante.

\subsection{5. Élimination des stratégies de négociation de la face}

Comme on l'a vu, les IV ont un rôle important dans la réalisation des stratégies de politesse; cela fait que leur élimination ait des conséquences sur les démarches employées par les locuteurs pour la négociation de la face.

\section{une faveur à l'autre:}

Ex. 21 (Mamma Roma)

VO: ahó : senti un po'::

VST: écoute.

91 Le rôle de l'IV est de retarder l'acte menaçant, ce que l'expression phatique « senti un po' » concourt à faire. L'acte de la requête est un acte menaçant pour la face négative du récepteur, car il constitue une intrusion dans son territoire (et pour sa face positive aussi au cas où il refuse); par conséquent, cet acte est généralement accompagné d'un élément de mitigation, à travers lequel le locuteur réduit son intrusion dans la face de l'autre et négocie sa propre face.

92 L'élimination de l'IV dans les sous-titres réduit la réalisation de la stratégie de politesse mise en acte par le locuteur dans l'énoncé en question. 
Voyons enfin un exemple d'élimination d'une IV non autonome:

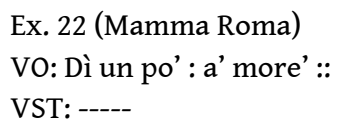

Dans ce cas aussi, l'acte menaçant de la requête est introduit par une pré-clause censée le retarder et le préparer, et qui permet au locuteur de négocier sa face à travers une double stratégie de politesse: négative, consistant en la réduction de son intrusion dans l'espace de l'autre, et réalisée à travers le phatique « dì un po'»); et positive, à travers l'emploi de l'appellatif, accompagné de l'IV, qui constitue un FFA.

Dans la V. ST non seulement l'IV mais aussi l'appellatif "moré » (abréviation de «moretto", qui signifie " petit brun ») et le phatique sont éliminés, ce qui annule toute stratégie de négociation de la face.

\section{Alternatives}

Une des possibilités pour compenser la perte des interjections vocatives nous est suggérée par Mamma Roma: il s'agit de l'emploi de l'interjection « hé »:

Ex. 23

VO: A' Sora Roma $\downarrow$

VST: Hé : Mamma Roma!

Dans le film de Pasolini cela arrive 9 fois. Le problème principal que ce type de solution pose est la difficulté de désambiguïsation à l'écrit, où l'on ne peut pas faire appui sur les données paraverbales: "hé" est un mot très flou, qui se prête à plusieurs interprétations et qui a besoin du contexte pour être désambigüisé.

Une autre possibilité pour récupérer l'élimination de ces particules (et, en particulier, de l'IV non autonome " $a$ '») est l'introduction de particules analogues qu'il est possible de trouver dans certaines variétés régionales, comme, par exemple, le «o'» du parler marseillais, dont on a parlé plus haut. Ce mot ale même sens, la même fréquence d'emploi dans la langue orale et la même fonction que la particule « a' »; en outre, s'agissant d'un mot dialectal, il a aussi le même statut diastratique.

Le problème, dans ce cas, est le fait que la traduction effectuée à travers le processus de la transposition dans une culture spécifique de la langue cible (domestication - cf. Venuti) pose toujours plusieurs difficultés, surtout au niveau pratique. Cela est vrai notamment dans les films, où les images se trouveraient, lors d'une solution de ce type, en contraste avec la langue des personnages.

Enfin, on peut envisager une solution peut-être plus adéquate dans l'emploi d'éléments relevant d'autres catégories linguistiques, qui rendent compte de la relation entre les interlocuteurs exprimée dans la version source. Dans l'exemple qui suit, cela est réalisé à travers l'emploi d'un adjectif qui n'existe pas dans la VO:

Ex. 24 (Mamma Roma)

VO: a' Nannare' ::

VST: Petite.

101 La dimension d'affection (l'appellatif "Nannarella", abrégé en "Nannarè"), perdue dans les ST à cause de l'élimination de l'appellatif, est récupérée ici à travers une autre catégorie linguistique (l'adjectif « petite »). 

d'adresse ayant une forte charge socioculturelle et interactionnelle. Leur élimination dans les ST, bien que justifiée par des raisons traductives et culturelles, comporte des changements dans la dimension interactionnelle des échanges: notamment, la réduction du jeu des distances sociales et des relationèmes, de la dimension ironique et sarcastique, des intentions agonales et conflictuelles ou bien de l'établissement d'une relation de camaraderie entre les interlocuteurs.

L'idée que dans les films les données visuelles et sonores compensent les manques dans les V. ST est très répandue; pourtant, il faut considérer que le spectateur ne peut pas 
saisir exactement toutes les images qui passent à l'écran vu qu'il doit lire les sous-titres; en outre, le décalage entre les images ou les sons et les sous-titres peut créer, chez les spectateurs, des effets de détachement.

À notre avis il est possible de compenser cette perte tout en respectant les contraintes du sous-titrage; notamment, la solution que nous proposons est la récupération de la force interpellative de ces particules et du sens (pragmatique, sociolinguistique, communicatif) qui lui est lié, à travers d'autres outils linguistiques qui ne font pas nécessairement partie de la même catégorie. Nous n'avons malheureusement pas ici l'espace pour pouvoir approfondir cette possibilité alternative, qui constituera l'objet d'études ultérieures; pour l'instant, ce qui est important, selon nous, et que le présent article a essayé de montrer, c'est de reconnaîtrela valeur linguistique de ces mots apparemment superflus afin de ne pas sous-estimer les nuances pragmatiques qu'elles véhiculent.

\section{BIBLIOGRAPHIE}

Barthes, R., (1972). Nouveaux essais critiques, Paris : Seuil.

Battaglia, S., (1960-2002). Grande dizionario della lingua italiana. Torino.

Bazzanella, C., (1994). Le facce del parlare, Firenze : La Nuova Italia.

Beeching, K. (2002). Gender, Politeness and Pragmatic Particles in French, Amsterdam/Philadelphia :

John Benjamins.

Blum-Kulka, S., (1989). House, J. and Kasper, G. (éd) Cross cultural pragmatics: Requests and apologies, Norwood, N.J.: Ablex.

Braun, F., (1998). Terms of address - Problems of patterns and usage in various languages and cultures, Amsterdam : Mouton de Gruyter.

Bres, J., (1995). "Hoù! Haa! Yrraa!: interjection, exclamation, actualisation", ds. Faits de langue 6, 81-91.

Charaudeau, P., (1992). Grammaire du sens et de l'expression, Hachette éducation.

Costa, G., (1981). "Pour une grammaire de l'interjection française", in Linguistica e Letteratura, 87-124.

Ducrot, O. et al., (1980). Les mots du discours, Paris : Minuit.

François, F., (1990). La communication inégale, Paris : Delachaux et Niestlé.

Gambier, Y., Gottlieb., H., (2001). (Multi)media translation, Philadelphia-Amsterdam: John Benjamins.

Gradus, (1984), Les procédés littéraires, Dupriez, 10/18.

Hall, E.T., (1971). La dimension cachée, Paris, Seuil.

Halliday, M.A.K., (1973). Explorations in the Functions of Language, London : Edward Arnold.

Hatim, B. et Mason, I., (1990). Discourse and the translator, Harlow: Longman. 
Heiss, C., Bollettieri Bosinelli, R. M., (1996). Traduzione multimediale per il cinema, la televisione e la scena, Bologna : Clueb.

Kerbrat-Orecchioni, K., (1990-1992-1994). Les interactions verbales, Paris, Armand Colin.

Nencioni, G., (1983). Tra grammatica e retorica, Torino : Einaudi.

Principato, A., (2000). Breve storia della lingua francese, Roma : Carocci.

Sacks, H., Schegloff, E. A., Jefferson, J., (1974). "A Simplest Systematics for the Organization of Turn-Taking for Conversation", in Language, Vol. 50, No. 4.

Serianni, L., (2000). Italiano, Milano : Garzanti.

Traverso, V., (1996). La conversation familière, analyse pragmatique des interactions, Lyon, PUL.

Venuti, L., (1995). The Translator's Invisibility: A History of Translation, London - New York: Routledge.

Vion, R., (1992). La communication verbale, Paris : Hachette.

Wierzbicka, A., (1985). "Different cultures, different languages, different speech acts", in Journal of Pragmatics 9 , pp. 145-161.

Wierzbicka, A., (1991). Cross-Cultural Pragmatics. The Semantics of Human Interaction, Berlin: Mouton de Gruyter.

\section{NOTES}

1. "[...] la référence linguistique du locuteur à son interlocuteur".

2. Proxémique: la distance physique entre les interlocuteurs.

3. Voir aussi, à ce propos, le concept de démocratisation de la langue dans Principato $(2000,141)$.

\section{RÉSUMÉS}

Le but de cette recherche est d'examiner la relation entre appellatif et interjection dans certaines formes d'interpellation italiennes dialectales au sein du dialogue filmique, et la façon dont ce phénomène est reproduit dans les respectives versions sous-titrées en français. Certaines particules, que nous appelons ici «interjections vocatives», sont des hybrides entre les catégories de l'adresse et de l'interjection, et doivent être étudiées comme des composantes du système de l'interpellation: bien qu'effaçables d'un point de vue syntaxique, elles sont porteuses de sens au niveau pragmatique-interactionnel.

Le corpus est formé de trois films italiens; à travers l'analyse d'une sélection d'exemples, nous montrerons les conséquences de l'élimination dans les sous-titres français de ces particules, généralement considérées comme superflues, et nous proposerons quelques alternatives tenant compte des contraintes de ce type de traduction.

The aim of the present paper is to examine the relationship between interjections and terms of address in certain dialectal forms of address in Italian film dialogs, and how this phenomenon is 
reproduced in the corresponding versions subtitled in French. Certain particles, called here "vocative interjections" are hybrids between the categories of the address and that of interjections, and they need to be studied as components of the system of address: even though they can be eliminated from a syntactic point of view, they have pragmatic and interactional sense.

The corpus is composed by three Italian films; through the analysis of a selection of examples, we will show the consequences of the elimination, in the correspondent French subtitles, of these particles which are usually considered as superfluous, and we will propose some alternative solutions, considering the practical limits of this type of translation.

INDEX

Mots-clés : interjections vocatives, système de l'adresse, sous-titrage, politesse

Keywords : vocative interjections, system of address, subtitling, politeness

\section{AUTEUR}

\section{CARLOTTA CINI}

Université de Pise (Département de Langues et Littératures Romanes)Membre du Do.Ri.F. (Centro di documentazione e di Ricerca per la didattica della lingua francese nell'Università italiana) 